\title{
Optical observations of southern planetary nebula candidates ${ }^{\star}$
}

\author{
G.C. Van de Steene ${ }^{1,3}$, K.C. Sahu ${ }^{2}$ and S.R. Pottasch ${ }^{3}$ \\ 1 European Southern Observatory, Casilla 19001, Santiago 19, Chile \\ 2 STScI, 3700 San Martin Drive, Baltimore, MD 21218, U.S.A. \\ 3 Kapteyn Astronomical Institute, P.O. Box 800, NL-9700 AV Groningen, The Netherlands
}

Received October 11, 1995; accepted April 11, 1996

\begin{abstract}
We present $\mathrm{H} \alpha+\left[\mathrm{N}_{\mathrm{II}}\right]$ images and low resolution spectra of 16 IRAS-selected, southern planetary nebula candidates previously detected in the radio continuum. The $\mathrm{H} \alpha+[\mathrm{N}$ II $]$ images are presented as finding charts. Contour plots are shown for the resolved planetary nebulae. From these images mean optical angular diameters were determined. Optical spectra show that these IRAS-selected and radio detected planetary nebula candidates are indeed planetary nebulae. The values for their extinction coefficient is generally very high. More than half of the planetary nebulae seem to be of low excitation, having central stars with an effective temperature of probably $\sim 60000 \mathrm{~K}$ or less.
\end{abstract}

Key words: planetary nebulae: general

\section{Introduction}

This is a continuation of the project to identify new planetary nebulae $(\mathrm{PN})$ applying the method as proposed by Pottasch et al. (1988). Unidentified IRAS sources were selected from the IRAS Point Source Catalog (PSC) on the basis of their far IR colors, which are typical of PN. Having selected the potential candidates, there are two ways of confirming that they are PN: first by observing the objects in the radio continuum, and second by means of optical spectra. Radio measurements are especially suitable for a first confirmation, because in this wavelength range the radiation is not attenuated by extinction. The detection of radio continuum emission provides strong evidence that the object is indeed a PN, because it shows the presence of ionized gas. To obtain sufficient positional accuracy for a correct identification, and to avoid confusion, these observations must be made with a synthesis array. This was successfully done for PN candidates inside the galactic bulge (Pottasch et al. 1988; Ratag et al. 1990; Ratag \& Pottasch 1991) and outside the galactic bulge (Van de Steene \& Pottasch 1993, 1994). Second, to confirm that these IRAS-selected and radio detected PN candidates are indeed PN, they should be observed in the optical. In this paper we present optical imaging and spectroscopy of radio detected PN candidates in the southern hemisphere (Van de Steene \& Pottasch 1994; hereafter Paper I).

Send offprint requests to: G.C. Van de Steene ${ }^{1}$

*Based on data acquired at the European Southern Observatory

\section{Selection of the planetary nebula candidates}

The sample candidates were selected from the IRAS PSC on the basis of their colors that are typical of PN. Only IRAS sources falling within the color box $F 12 / F 25 \leq 0.35$ and $F 25 / F 60 \geq 0.35$ were chosen, to avoid confusion with galaxies and HII regions having colors just outside this box ( $F 12, F 25$, and $F 60$ refer to the flux in the IRAS wavelength bands centered at 12,25 , and $60 \mu \mathrm{m}$ respectively). The sample sources have good flux values (quality 2 or 3 ) at 12,25 and $60 \mu \mathrm{m}$. The PN candidates are outside the galactic bulge, farther than 15 degrees in longitude from the galactic center. The list of these IRAS-selected and radio observed PN candidates was presented in Paper I.

\section{Observations}

\subsection{Imaging}

Images of all IRAS-selected PN candidates were taken through an $\mathrm{H} \alpha+[\mathrm{N} \mathrm{II}]$ filter with the Dutch $0.9 \mathrm{~m}$ telescope of the European Southern Observatory at La Silla (Chile) during January and May 1993. The main goal of taking the images was to identify the PN candidates, and then use the images as finding charts for optical spectroscopy. We also wanted to determine the mean optical angular diameters of the PN, in order to compare them with the ones obtained from the radio continuum images.

In January the candidates were observed using the RCA 5049-1-8 CCD of $512 \times 320$ pixels having a pixelsize of 0.449 . The filters used were the $\mathrm{H} \alpha+[\mathrm{N}$ II] standard 
Table 1. Imaging observation log of detected PN candidates

\begin{tabular}{llll}
\hline Object & Observer & Date & Time \\
\hline $0835-4027$ & Snellen & $21 / 01 / 93$ & $10 \mathrm{~min}$ \\
$0857-5011$ & Snellen & $22 / 01 / 93$ & $15 \mathrm{~min}$ \\
$0936-5413$ & Snellen & $22 / 01 / 93$ & $15 \mathrm{~min}$ \\
$1002-5553$ & Snellen & $22 / 01 / 93$ & $15 \mathrm{~min}$ \\
$1011-5640$ & Snellen & $21 / 01 / 93$ & $15 \mathrm{~min}$ \\
$1200-5333$ & Snellen & $21 / 01 / 93$ & $15 \mathrm{~min}$ \\
$1231-6401$ & Snellen & $21 / 01 / 93$ & $15 \mathrm{~min}$ \\
$1412-5947$ & Snellen & $22 / 01 / 93$ & $15 \mathrm{~min}$ \\
$1417-5824$ & Snellen & $22 / 01 / 93$ & $15 \mathrm{~min}$ \\
$1434-5858$ & Snellen & $24 / 01 / 93$ & $10 \mathrm{~min}$ \\
$1557-5445$ & Vd Steene & $20 / 05 / 93$ & $4 \mathrm{~min}$ \\
$1600-5041$ & Vd Steene & $21 / 05 / 93$ & $15 \mathrm{~min}$ \\
$1611-4504$ & Vd Steene & $21 / 05 / 93$ & $14 \mathrm{~min}$ \\
$1633-4807$ & Vd Steene & $22 / 05 / 93$ & $20 \mathrm{~min}$ \\
$1652-4341$ & Vd Steene & $22 / 05 / 93$ & $20 \mathrm{~min}$ \\
$1708-4227$ & Vd Steene & $21 / 05 / 93$ & $15 \mathrm{~min}$ \\
\hline
\end{tabular}

ESO filter Nr. 387 with a FWHM of $81.8 \AA$ centered at $\lambda 6568.5 \AA$ and the ESO filter broadband filter Nr. 420 filter with a FWHM of $116.9 \AA$ centered at $\lambda 5443.6 \AA$. In May we used the same filters, but the CCD TEK512 Nr. 33 was installed, which has $512 \times 512$ pixels of $0{ }^{\prime \prime} 44$.

The images were reduced using standard procedures in the IRAF reduction package as described by Massey (1992). Afterwards the images were searched for the presence of a PN near the center of the field using the following procedure: each $\mathrm{H} \alpha+[\mathrm{N}$ II] image was divided by the continuum one, and all three images were blinked.

Table 1 gives an observation $\log$ for the the $\mathrm{H} \alpha+[\mathrm{N}$ II] images in which a PN was identified. The first column indicates the object, the second column the observer's name, the third column the observation date, and the fourth column the exposure time of the images.

\subsection{Spectroscopy}

The name of the PN candidate, the date, the exposure time and the orientation of the slit are listed in Table 2. Spectra were taken at the ESO $1.52 \mathrm{~m}$ telescope with the Boller \& Chivens spectrograph in March and May 1993. We used grating number 2 which has $300 \mathrm{gr} / \mathrm{mm}$. The detector used was a Ford Aerospace 2048L pixel CCD with a pixel size of $15 \mu \mathrm{m}$. The dispersion at the detector plane is $253 \AA$ per mm, which corresponds to $3.8 \AA$ per pixel. The spectral range was from $\sim 3500$ to $10500 \AA$. The spectra were reduced using standard procedures of the reduction package MIDAS. The wavelength calibration was done using a HeAr calibration lamp. For deriving the response curve of the detector, 2 to 3 standard stars were observed each night. The flux determinations were done using the gaussian fitting and deblending commands of
Table 2. Spectroscopy observation log

\begin{tabular}{lllll}
\hline Object & Observer & Date & Time & slit p.a. \\
\hline $0835-4027$ & Sahu/Manchado & $12 / 03 / 93$ & $1 \mathrm{~h}$ & -90 \\
$0857-5011$ & Sahu/Manchado & $12 / 03 / 93$ & $45 \mathrm{~min}$ & -90 \\
$0936-5413$ & Sahu/Manchado & $13 / 03 / 93$ & $1 \mathrm{~h}$ & +80 \\
$1011-5640$ & Sahu/Manchado & $12 / 03 / 93$ & $1 \mathrm{~h}$ & -90 \\
$1200-5333$ & Sahu/Manchado & $12 / 03 / 93$ & $1 \mathrm{~h}$ & -90 \\
$1231-6401$ & Vd Steene & $24 / 05 / 93$ & $1 \mathrm{~h}$ & -90 \\
$1412-5947$ & Vd Steene & $24 / 05 / 93$ & $1 \mathrm{~h}$ & -90 \\
$1417-5824$ & Vd Steene & $25 / 05 / 93$ & $1 \mathrm{~h}$ & -1.3 \\
$1434-5858$ & Vd Steene & $25 / 05 / 93$ & $1 \mathrm{~h}$ & -89.6 \\
$1557-5445$ & Vd Steene & $24 / 05 / 93$ & $1 \mathrm{~h}$ & -90 \\
$1600-5041$ & Vd Steene & $25 / 05 / 93$ & $45 \mathrm{~min}$ & -86.6 \\
$1611-4504$ & Sahu & $23 / 08 / 92$ & $5 \mathrm{~min}$ & -90 \\
$1633-4807$ & Vd Steene & $26 / 05 / 93$ & $1 \mathrm{~h}$ & -21.2 \\
$1652-4341$ & Vd Steene & $24 / 05 / 93$ & $1 \mathrm{~h}$ & -90 \\
$1708-4227$ & Vd Steene & $25 / 05 / 93$ & $1 \mathrm{~h}$ & -89.6 \\
\hline
\end{tabular}

the splot program in iraf, which compute a non-linear least-squares Gaussian fit to the line(s). Usually, lines less than $20 \AA$ apart were deblended.

\section{Discussion}

\subsection{Imaging}

The images of all identified PN candidates are presented in appendix A as finding charts. If the PN was resolved, its contour plot is shown in Appendix B.

Table 3. Comparison between optical and radio angular diameters

\begin{tabular}{|c|c|c|c|c|c|c|}
\hline \multirow[t]{2}{*}{ Name } & $l$ & $b$ & \multirow{2}{*}{$\theta_{\mathrm{PSF}}$} & \multirow{2}{*}{$\theta_{\mathrm{FWHM}}$} & \multirow{2}{*}{$\theta_{\mathrm{Str}}$} & \multirow{2}{*}{$\theta_{\text {radio }}$} \\
\hline & $\mathrm{O}$ & $\mathrm{O}$ & & & & \\
\hline $0835-4027$ & 260.15 & +0.25 & 1.8 & ext. & 11.2 & 10.8 \\
\hline $0857-5011$ & 270.12 & -2.95 & 1.3 & 2.3 & 4.2 & 3.0 \\
\hline $0936-5413$ & 277.15 & -1.57 & 1.3 & $<1.3$ & $<2.3$ & 2.0 \\
\hline $1002-5553$ & 281.18 & -0.48 & 1.2 & 0.6 & 1.1 & $<3.1$ \\
\hline $1011-5640$ & 281.61 & -0.43 & 1.1 & 2.1 & 3.7 & $<3.4$ \\
\hline $1231-6401$ & 301.11 & -1.49 & 1.0 & ext. & 3.2 & 1.2: \\
\hline $1412-5947$ & 313.30 & +1.13 & 1.3 & ext. & 2.6 & $<4.1$ \\
\hline $1417-5824$ & 314.42 & +2.21 & 1.3 & ext. & $11.2 \times 4.7$ & $<4.1$ \\
\hline $1434-5858$ & 316.25 & +0.88 & 1.6 & 0.6 & 1.0 & $<9.0$ \\
\hline $1557-5445$ & 328.07 & -1.60 & 1.8 & $<1.8$ & $<3.2$ & 1.8 \\
\hline $1600-5041$ & 331.03 & +1.21 & 2.1 & $<2.1$ & $<3.8$ & 2.7 \\
\hline $1611-4504$ & 336.18 & +4.13 & 1.9 & & 7.0 & 6.1 \\
\hline $1633-4807$ & 336.64 & -0.70 & 2.2 & 0.8: & 1.4: & 2.9 \\
\hline $1652-4341$ & 342.23 & -0.37 & 2.9 & 2.8 & 5.0 & 4.4 \\
\hline $1708-4227$ & 344.98 & -1.91 & 1.5 & 1.2 & 2.1 & 2.1 \\
\hline
\end{tabular}

\subsubsection{Positional accuracy}

We tried to obtain an accurate optical position of the PN using the HST guide star catalog and the package FINDER in IRAF, but the imaged fields were too small 
to contain enough guide stars to obtain optical positions with better accuracy than the radio ones. The best we could do was to verify in some cases that the optical positions were comparable to the radio ones, which are in turn close enough to the IRAS source to be associated with it. We think that all optically identified PN are indeed associated with the radio and IR sources. The accuracy of the radio positions is expected to be better than $1^{\prime \prime}$ in right ascension and declination for all sources.

\subsubsection{Angular diameters}

The point-spread function is well approximated by a gaussian with a FWHM of $\theta_{\mathrm{PSF}}$, derived from the second moments of the profiles of field stars and given in Col. 4 of Table 3. We also determined in this way the FWHM of the PN, $\theta_{\mathrm{PN}}$, and then calculated its deconvolved FWHM (given in Col. 5 of Table 3 ), according to $\theta_{\mathrm{FWHM}}=$ $\sqrt{\theta_{\mathrm{PN}}^{2}-\theta_{\mathrm{PSF}}^{2}}$. However a gaussian profile is a rather poor description of the intensity distribution of a PN. In the case of a partially resolved object, the best approach is to calculate $\theta_{\text {FWHM }}$ and then apply a correction factor based on an assumed intensity distribution to obtain the true Strömgren angular diameter of the PN (Panagia \& Walmsley 1978; Bedding \& Zijlstra 1994). The Strömgren angular diameters, $\theta_{\mathrm{Str}}$ listed in Col. 6 of Table 3 were calculated assuming that the ratio of the inner radius to the Strömgren radius is equal to a half. From the resulting intenstity distribution we determined that $\theta_{\text {FWHM }}$ should be multiplied $\Omega$ with a correction factor of 1.8 to obtain the Strömgren radius (van Hoof 1996, in preparation).

For PN whose appearance is stellar an upper-limit is given; the angular diameters of the ones for which the $\theta_{\text {FWHM }}$ is slightly larger than stellar are marked with a colon. The angular sizes of the well resolved PN were measured in RA and DEC from the lowest contour in the plots presented in Appendix B. Then the geometric mean diameter was calculated and listed in Table 3.

For comparison the radio diameters are given in Col. 7 of Table 3 . The radio angular diameters may be less accurate due to the relatively small sizes of the PN compared to the beam, and the lack of $u v$-coverage. After all the goal of the radio continuum observations had been to try and detect the PN candidates, not to determine accurate angular diameters.

\subsubsection{Detection statistics}

The PN candidates $1240-6219$ and $1510-5754$ were detected in the radio continuum but not in $\mathrm{H} \alpha$. If they are indeed PN, they are probably not detected because of large extinction (Kistiakowsky \& Helfland 1993).

Another reason could be that they are extra-galactic background sources. However, based on source counts at
$6 \mathrm{~cm}$ (Donnely et al. 1987), one would expect $\sim 5 \%$, i.e. at most one, of the radio detections to be a background source. Besides the background galaxy 1200-5333 which we detected, is clearly seen in $\mathrm{H} \alpha$.

The observers Sahu/Manchado didn't have the $\mathrm{H} \alpha \mathrm{im}-$ ages at their disposal, but by offsetting the telescope from a bright star to the accurate radio position, they managed to obtain some spectra. This offset method for finding the PN works only if the field is not too crowded and the telescope pointing is good. Sahu \& Manchado could not identify $1633-4807$ for instance.

\subsection{Spectroscopic observations}

Table 4. Values for logarithmic extinction $A_{V}(\mathrm{mag})=2.1$ $c(\mathrm{H} \beta)=3.1 c(\mathrm{H} \alpha)$

\begin{tabular}{llllll}
\hline Name & $c_{\mathrm{H} \beta}$ & $c_{\mathrm{H} \alpha}$ & $\begin{array}{l}A_{V} \\
\mathrm{mag}\end{array}$ & $\begin{array}{l}\text { Excitation } \\
\text { Class }\end{array}$ & {$[\mathrm{S} \mathrm{III}] /[\mathrm{O}$ III $]$} \\
\hline $0835-4027$ & 3.7 & & 7.8 & 2.6 & 0.6 \\
$0857-5011$ & 2.7 & & 5.7 & 6.0 & 0.02 \\
$0936-5413$ & 2.8 & & 5.9 & 1.2 & 0.17 \\
$1011-5640$ & - & 3.4 & 10.5 & 5.9 & - \\
$1231-6401$ & 1.8 & & 3.8 & 6.6 & 0.12 \\
$1412-5947$ & - & 3.0 & 9.3 & 5.3 & 50.6 \\
$1417-5824$ & 4.0 & & 8.4 & 6.5 & 2.7 \\
$1434-5858$ & - & 2.5 & 7.8 & 2.1 & $19.9:$ \\
$1557-5445$ & 4.0 & & 8.4 & 0.1 & 1.8 \\
$1600-5041$ & - & 3.9 & 12.1 & - & - \\
$1611-4504$ & 2.6 & & 5.5 & 6.1 & - \\
$1633-4807$ & - & 2.7 & 8.4 & - & - \\
$1652-4341$ & - & 3.4 & 10.5 & 3.5 & 12.7 \\
$1708-4227$ & 0.9 & & 1.9 & - & - \\
\hline
\end{tabular}

The spectra of the PN candidates detected in $\mathrm{H} \alpha$ are shown in Appendix C. The Table with line-identifications and line-ratios normalized to $\mathrm{H} \beta$, or $[\mathrm{O}$ III $] \lambda 5007, \mathrm{H} \alpha$ depending upon which line was available and best determined, are presented in Appendix D. The line-fluxes were corrected for interstellar extinction using the extinction law from Seaton (1979).

This interstellar extinction law and the observed $\mathrm{H} \alpha / \mathrm{H} \beta$ ratio, when compared to the recombination value of 2.85 (Aller 1984), gives a logarithmic extinction at $\mathrm{H} \beta$ : $c(\mathrm{H} \beta)=3.096 \log (\mathrm{H} \alpha /(\mathrm{H} \beta 2.85))$

corresponding to a visual extinction of $A_{V}=2.1 c(\mathrm{H} \beta)$. For PN without detectable $\mathrm{H} \beta$ emission, we used the previous expression $c(\mathrm{H} \beta)$, together with the equation by Pottasch (1984) based on the radio flux:

$c(\mathrm{H} \beta)=\log \left(S_{6 \mathrm{~cm}} /(3.67 \mathrm{H} \beta)\right)$,

to derive the logarithmic extinction value at $\mathrm{H} \alpha$, corresponding to a visual extinction of $A_{V}=3.1 c(\mathrm{H} \alpha)$. However this is a rough estimate for the extinction, because not all nights were photometric and for the larger nebulae, some flux will have been missed due to the $2^{\prime \prime}$ slit 
width. The values for extinction based on $\mathrm{H} \alpha$ and radio flux are therefore likely to be less accurate. The values for the logarithmic extinctions are presented in Table 4. The fact that no $\mathrm{H} \beta$ emission was seen, is in itself a clear indication for a high extinction, with $A_{V}$ larger than $\sim 8.0$. While the values for $A_{V}$ of optically known PN are in the range 0.04 to 4.8 (Osterbrock 1989) we see that for only 2 of the PN in our sample, $A_{V}$ is smaller than 4.8.

Usually the strongest emission lines in the spectra of optically known $\mathrm{PN}$ are the $[\mathrm{O} \mathrm{III}] \lambda 5007$ and $\mathrm{H} \alpha$ emission lines. However it was noticed that for PN exhibiting large extinction, discovered on the basis of IRAS and radio continuum measurements, the $[\mathrm{S} \mathrm{III}] \lambda 9532$ emission line often appears as the strongest line in the PN spectrum. Kistiakowsky \& Helfland (1993) predicted that for galactic $\mathrm{PN}$ the $[\mathrm{S} \mathrm{III}] \lambda 9532$ appears stronger than $[\mathrm{O}$ III $] \lambda 5007$ whenever the visual extinction exceeds 3 magnitudes. For 10 of our 14 spectra the $[\mathrm{S} \mathrm{III}] \lambda 9532$ seems clearly stronger than $[\mathrm{O}$ III $] \lambda 5007$, and for 8 of these 10 the $[\mathrm{S} \mathrm{III]} \lambda 9532$ line appears even stronger than $\mathrm{H} \alpha$. The former seems to occur whenever the visual extinction is larger than about 5.5 magnitudes and the latter whenever the visual extinction is larger than 8 magnitudes. Three PN have such a high extinction that both the $\mathrm{H} \beta$ and [O III] emission lines are absent.

After correction for extinction, the excitation classes of the PN calculated according to Dopita \& Meatheringham (1990), are given in Col. 5 of Table 4. The relationship between excitation class and stellar effective temperature is analogous to the relationship between stellar spectral type and effective temperature in the case of stars. An excitation class higher than 5 means that He II $\lambda 4686$ was observed in the spectrum and that the stellar temperature is higher $\sim 60000 \mathrm{~K}$. From Table 4 we see that about half the number of PN are of low excitation.

\section{Notes on individual objects}

We searched the literature using the Simbad database to collect up to date information about the individual PN. Half of them were listed in Preite-Martinez (1988) as possible PN candidates on the basis of their IRAS colors. Our objects are not listed in the Strasbourg-ESO catalogue of galactic PN.

0835-4027: This PN was oberved at $843 \mathrm{MHz}$ with the Molongolo Synthesis Telescope by Whiteoak (1992) who determined a radio flux of $130 \mathrm{mJy}$. The fact that this value is slightly higher than ours could be due to the fact that the MOST fields were not cleaned and this radiotelescope had a 10 times larger beam.

1002-5553: This PN candidate was observed in CO by Loup et al. (1990), but the spectrum was affected by interstellar contamination. No spectrum was obtained for this PN candidate. We have insufficient data to confirm this object as PN.
Gal 1200-5333: It was already suggested in Paper I that, based on its IRAS colors, this source could be a galaxy. The $\mathrm{H} \alpha$ image shows that this is not a PN and the emission lines in the spectrum are redshifted by about $190 \AA$.

1231-6401: In the $\mathrm{H} \alpha$ image taken with the Dutch $0.90 \mathrm{~m}$ telescope the object appears to be asymmetric, a bit elongated, and surrounded by a box-like much larger fainter halo. This PN seems elongated and asymmetric, but higher spatial resolution is needed to investigate its morphology. Silva et al. (1993) searched for hydroxyl in this cold IRAS source but didn't detect any. This nebula was observed, and detected, in the $\mathrm{CO}(2-1)$ line with the SEST, and was seen to have an intensity of $5.8 \mathrm{~K}-\mathrm{km} / \mathrm{s}$ and an expansion velocity of $25 \mathrm{~km} / \mathrm{s}$ (R. Sahai private communication). However the emission lines are generally broader than for the other new PN in our sample. they may be slightly resolved, which is indicative of a large expansion velocity of the gas. The co-existence of a strong [O I] line with a strong He II line is puzzling. No consistent excitation class could be determined from the He II and $[\mathrm{O}$ III $] \lambda 5007$ line. The spectrum shows iron lines though many of these are in blends. We identified $[\mathrm{Fe}$ III $] \lambda 5270$ and $[\mathrm{Fe}$ VIII $] \lambda 6087$. One would expect iron to be depleted in grains in PN, though iron has been seen in high resolution spectra of other PN such as IC 2165 (Huyng 1994) and IC 4997 (Hyung et al. 1994). The forbidden iron emission lines probably originate in the higher density regions of the nebula.

1412-5947: This PN clearly has an elongated morphology, but an image of higher spatial resolution is needed to investigate its, appearent bipolar, structure.

1417-5824: This PN is clearly bipolar. The spectrum was taken along the major axis and some of the stellar continuum is visible in the spectrum.

1510-5754: Silva et al. (1993) searched for hydroxyl in this cold IRAS source but didn't detect any. Nor did Loup et al. (1990) find any CO. We could not identify this radio source in $\mathrm{H} \alpha$, neither did we obtain a spectrum. It probably is very extinct and its PN nature should be further investigated at IR wavelengths.

1557-5445: This PN candidate has the second lowest excitation class: it shows $\mathrm{H} \beta$ emission, but weak $[\mathrm{O}$ III $] \lambda 5007$, so that its stellar temperature would be less than $30000 \mathrm{~K}$. Because its IRAS colors are atypical for a compact $\mathrm{H}$ II region (Paper I), this object is likely to be a very young $\mathrm{PN}$, worthy of monitoring.

1600-5041: This PN seems to have the highest extinction of all, and consequently only a few emission lines at longer wavelengths are visible in the spectrum.

1611-4504: This PN shows a double peak at its center. However an image at higher resolution is needed to confirm this feature.

1708-4227: This PN has the lowest extinction and the lowest excitation, having a stellar temperature likely 
below $30000 \mathrm{~K}$. The stellar continuum and some stellar absorption lines are visible in the spectrum. This object seems to be very young PN, worthy of further monitoring.

\section{Conclusions}

Almost all 16 PN candidates selected from the IRAS PSC based on their colors typical for $\mathrm{PN}$, detected in the radio continuum, were confirmed as PN by optical spectroscopy. Most of these IRAS PN are heavily extinct having an average $A_{V}$ of $7 \mathrm{mag}$. Large extinction may be the reason why some of the radio detected $\mathrm{PN}$ candidates have gone undetected in $\mathrm{H} \alpha$. About half of the PN seem to be of low excitation, having a stellar effective temperature lower than $\sim 60000 \mathrm{~K}$. Careful modeling has been done in order to consistently determine the physical parameters of the PN with good optical spectra. The modeling and the results will be discussed in forthcoming papers (van Hoof \& Van de Steene 1996 and Van de Steene \& van Hoof 1995, in preparation; Van de Steene 1996).

Acknowledgements. G.C. Van de Steene acknowledges support from the Netherlands Foundation for Research in Astronomy (ASTRON) under grant number 782-372-035. This research has made use of the Simbad database, operated at CDS, Strasbourg, France. We thank P.A.M. van Hoof for critical reading of the manuscript. We thank E. Snellen and A. Manchado for doing some of the observations.

\section{References}

Acker A., Marcout J., Ochsenbein F., Stenholm Tylenda R., 1992, Strasbourg-ESO catalogue of galactic planetary nebulae, ESO
Aller L.H., 1984, Physics of Thermal Gaseous Nebulae. D. Reidel, Dordrecht

Bedding T.R., Zijlstra A.A., 1994, A\&A 283, 955

Donnely R.H., Partridge R.B., Windhorst R.A., 1987, ApJ 321, 94

Dopita M.A., Meatheringham S.J., 1990, ApJ 357, 140

Hyung S., 1994, ApJS 90, 119

Huyng S., Aller L.H., Feibelman W.A., 1994, ApJS 93, 465

Kistiakowsky V., Helfland D.J., 1993, AJ 105, 2199

Loup C., Forveille T., Nyman L.A., Omont A., 1990, A\&A 227, L29

Massey P., 1992, “A User's Guide to CCD Reductions with IRAF", IRAF help package

Osterbrock D.E., 1989, 'Astrophysics of Gaseous Nebulae and Active Galactic Nuclei', Ch. 7, University Science, California

Panagia N., Walmsley C.M., 1978, A\&A 70, 411

Pottasch S.R., 1984, "Planetary Nebulae". D. Reidel, Dordrecht, p. 93

Pottasch S.R., Bignell C., Olling R., Zijlstra A.A., 1988, A\&A 205, 248

Preite-Martinez A., 1988, A\&AS 76, 317

Ratag M.A., Pottasch S.R., Zijlstra A.A., Menzies J., 1990, A\&A 233, 181

Ratag M.A., Pottasch S.R., 1991, A\&AS 91, 481

Seaton M.J., 1979, MNRAS 187, 73

Silva A.M., Azcárate I.N., Pöppel W.G.L., Likkel L., 1993, A\&A 275,510

Van de Steene G.C., 1995, PhD thesis, University of Groningen

Van de Steene G.C., Pottasch S.R., 1993, A\&A 274, 895 (Paper I)

Van de Steene G.C., Pottasch S.R., 1995, A\&A 299, 238

Whiteoak J.B.Z., 1992, A\&A 262, 251 


\section{A. $\mathbf{H} \boldsymbol{\alpha}+\left[\mathrm{N}_{\mathrm{II}}\right]$ images}

In this appendix all $\mathrm{H} \alpha+[\mathrm{NII}]$ images in which a $\mathrm{PN}$ was identified are presented as finding charts. The $\mathrm{PN}$ is indicated by a box. North is at the top and east is to the right. For Figs. 1 to 10 the field of view is $2 \times 2$ arcminutes. For Figs. 11 to 16 the field of view is $3.5 \times 3.5$ arcminutes.

Fig. 1. 0835-4027

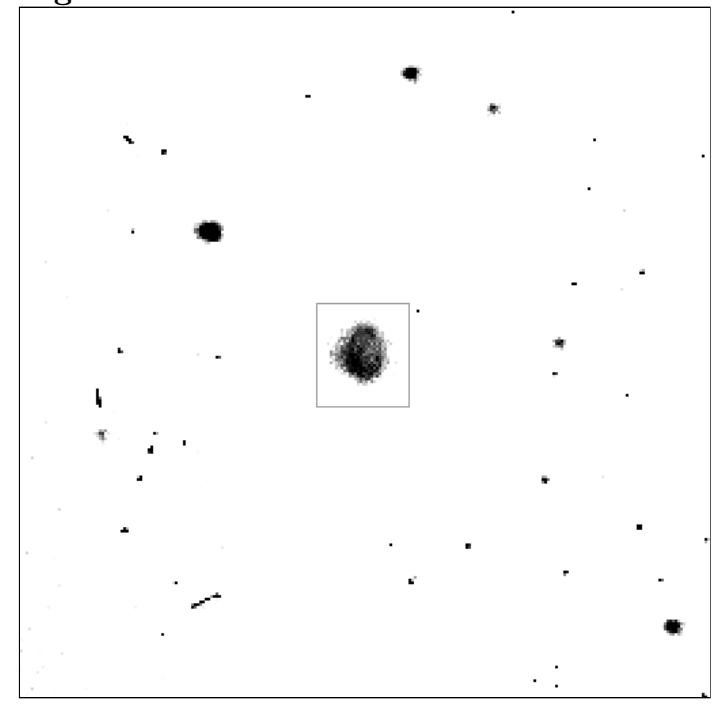

Fig. 2. 0857-5011

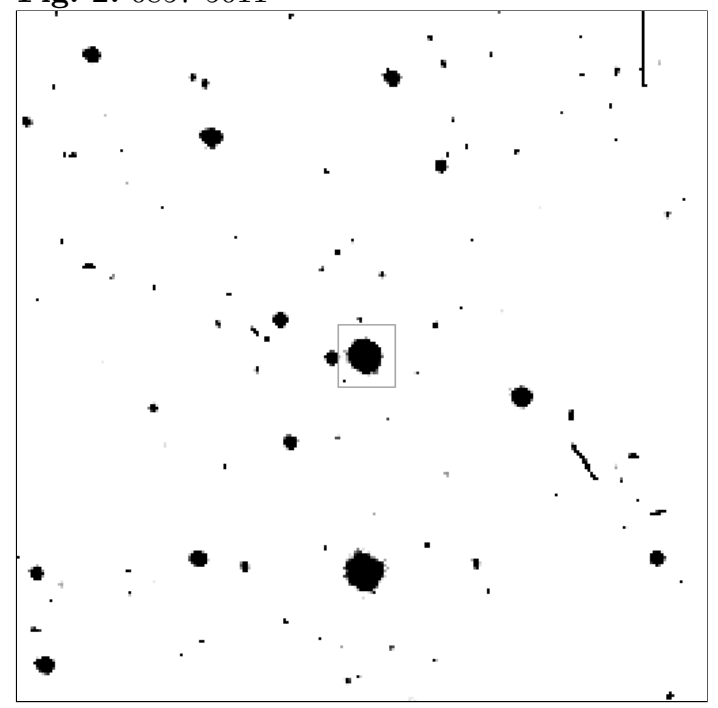

Fig. 3. 0936-5413

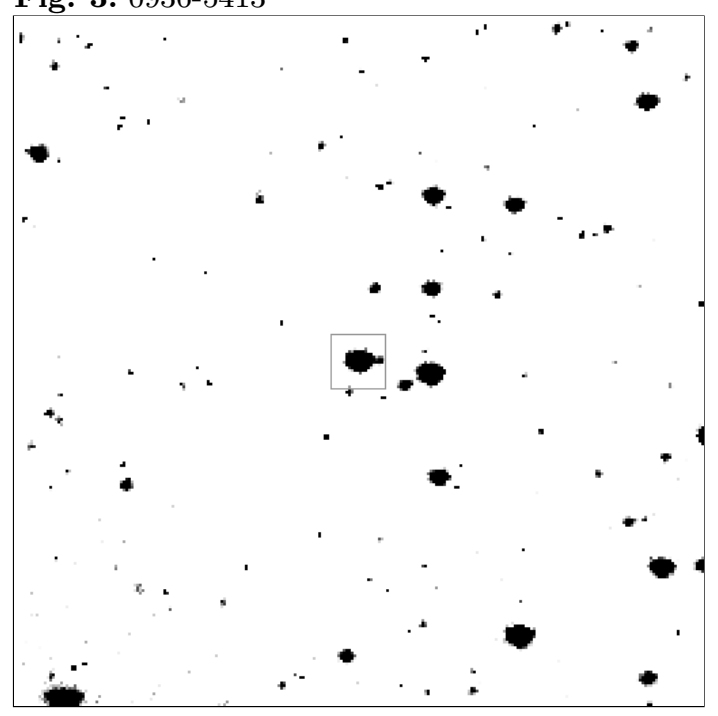

Fig. 4. 1002-5553

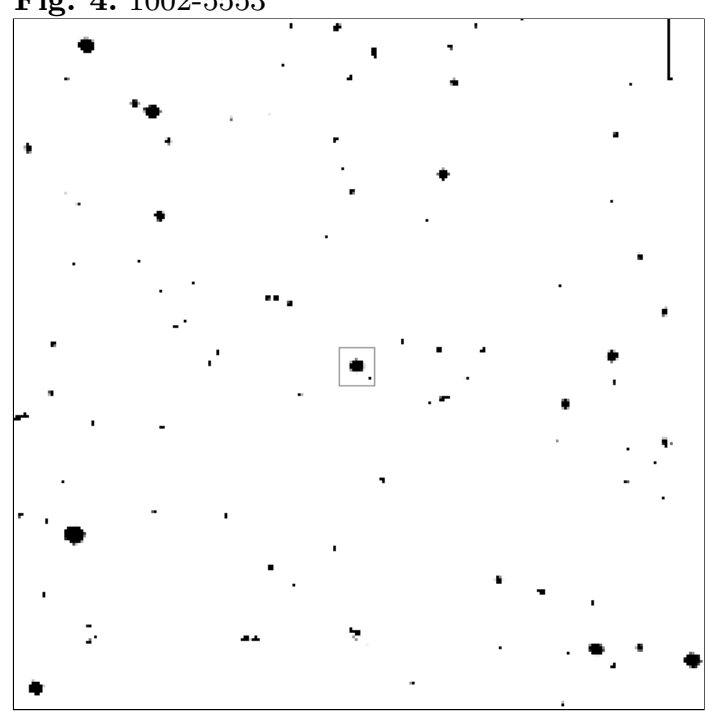

Fig. 5. 1011-5640

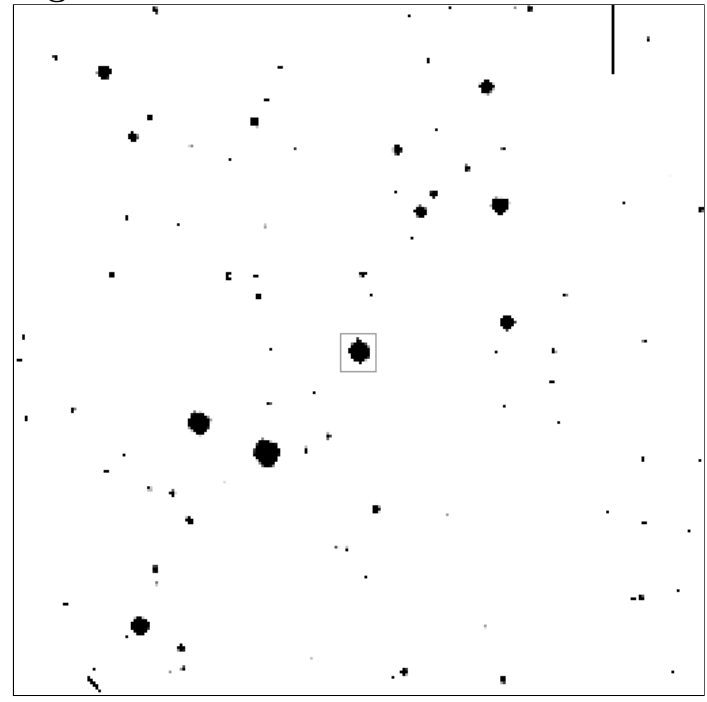


Fig. 6. 1200-5333

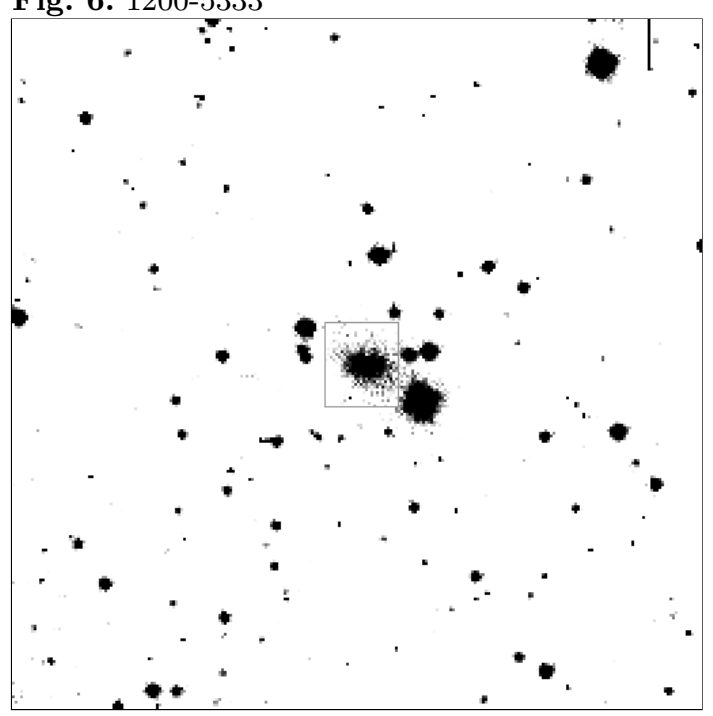

Fig. 7. 1231-6401

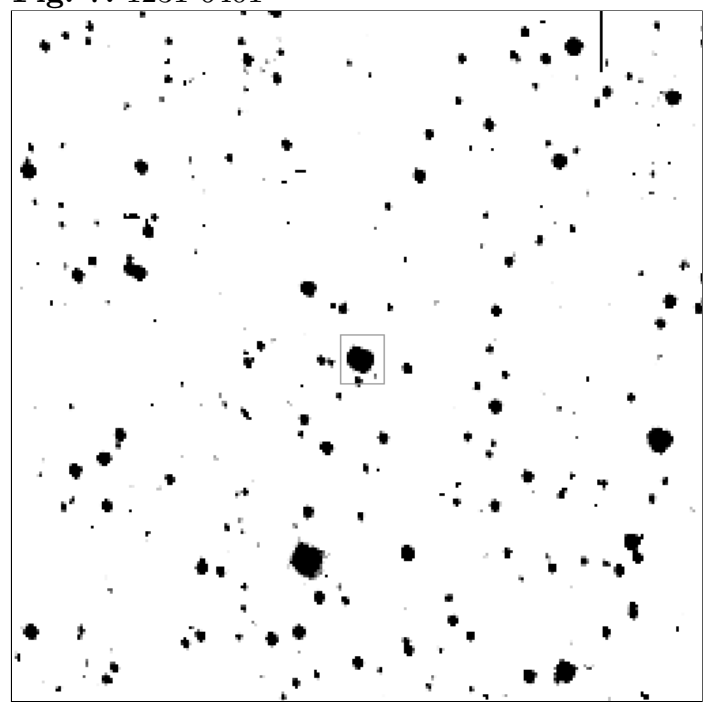

Fig. 8. 1412-5947

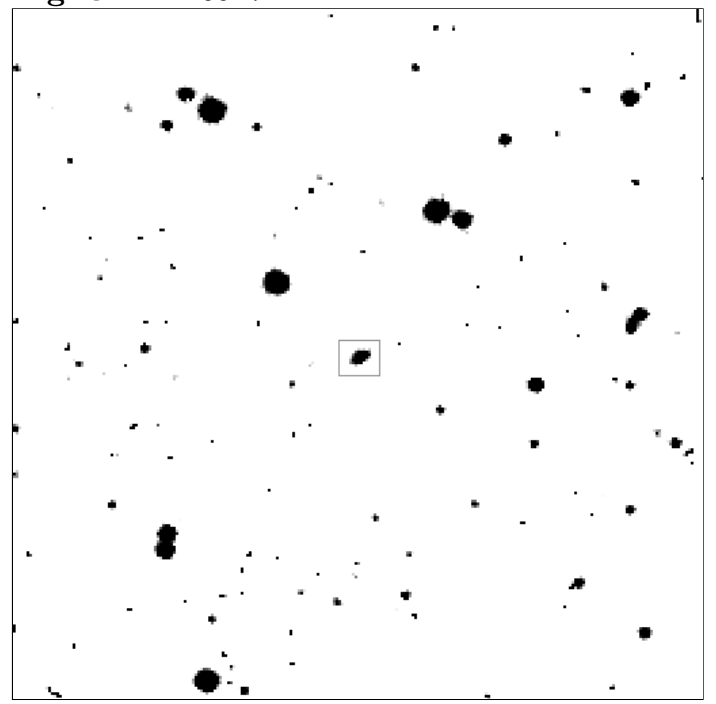

Fig. 9. 1417-5824

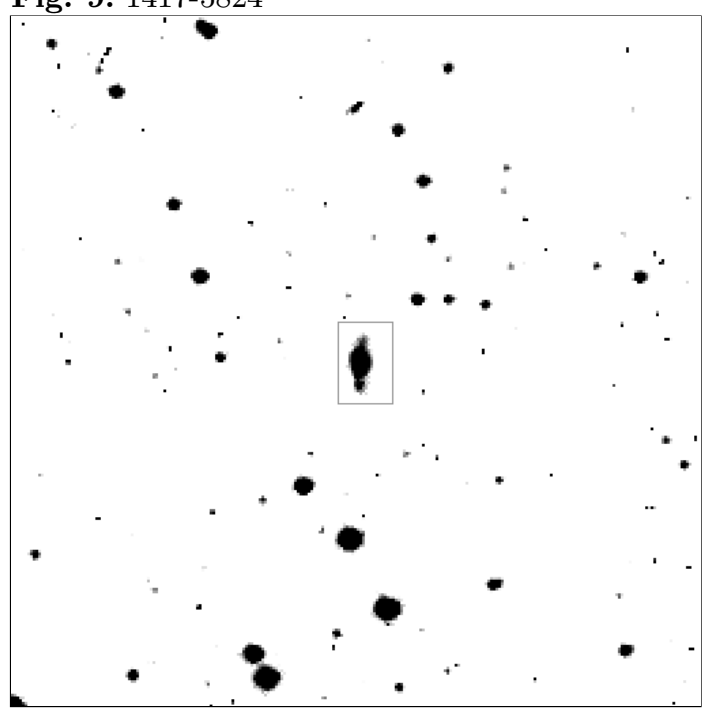

Fig. 10. 1434-5858

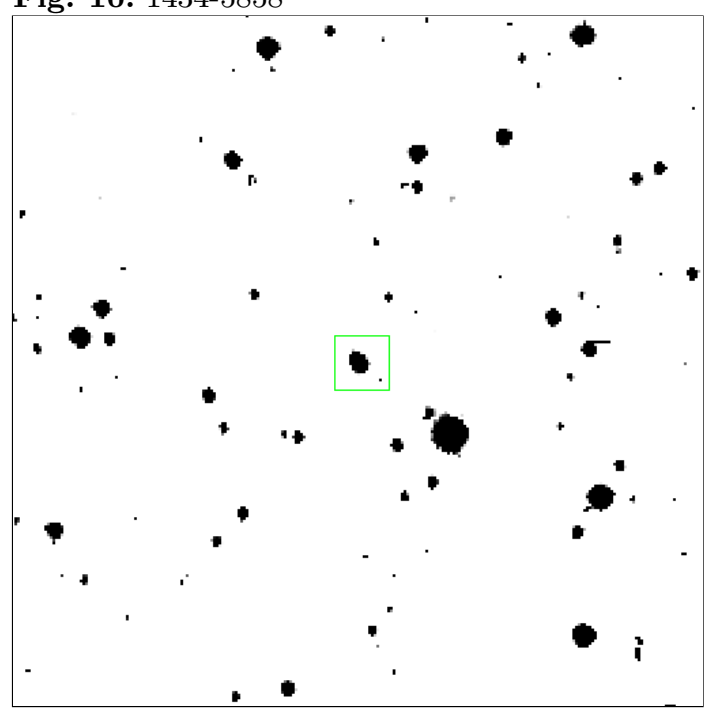

Fig. 11. 1557-5445

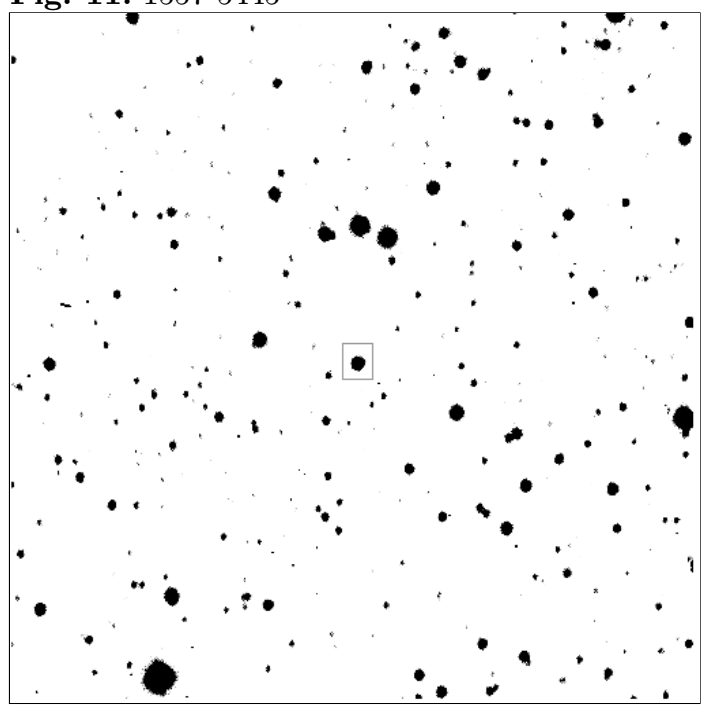


Fig. 12. 1600-5041

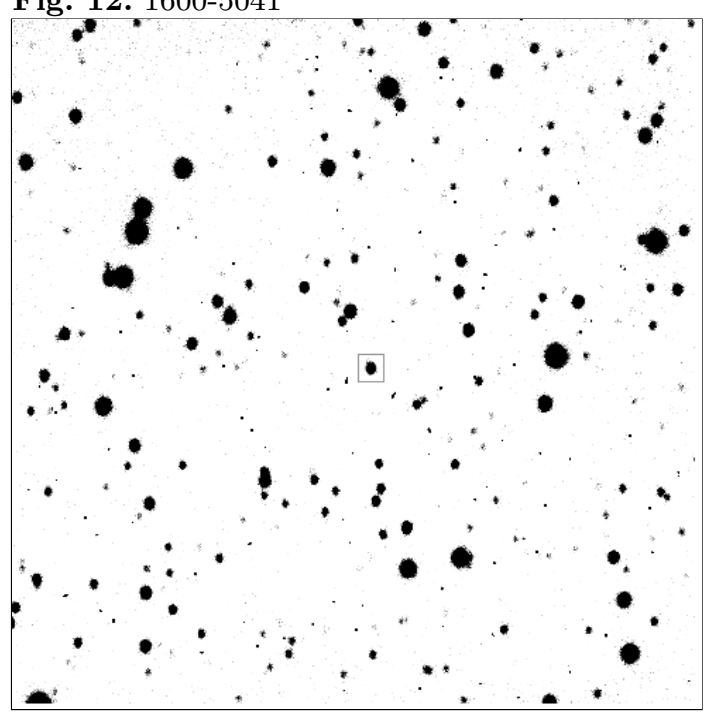

Fig. 13. 1611-4504

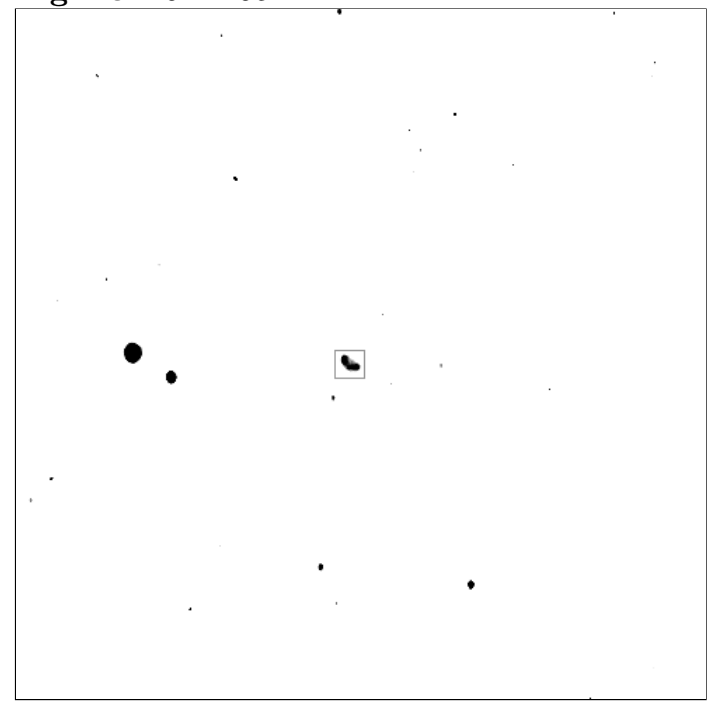

Fig. 14. 1633-4807

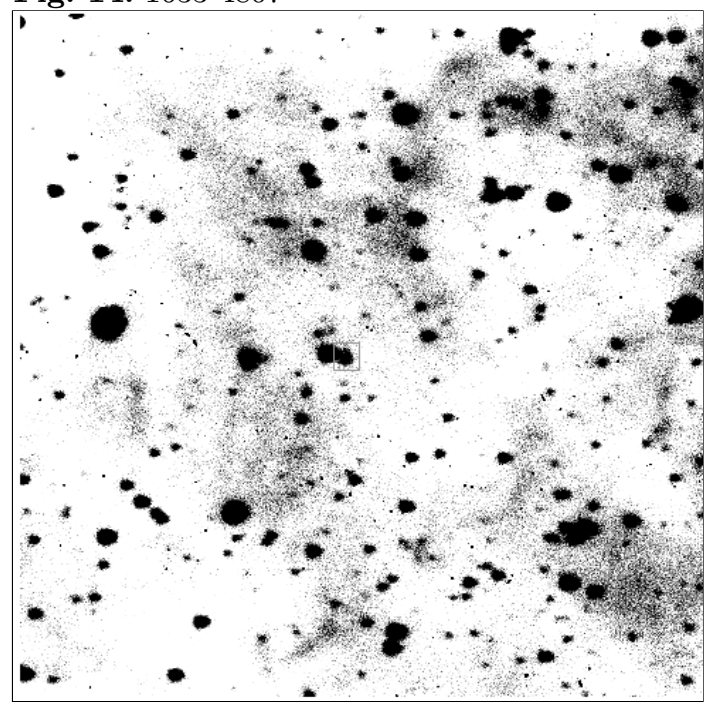

Fig. 15. 1652-4341

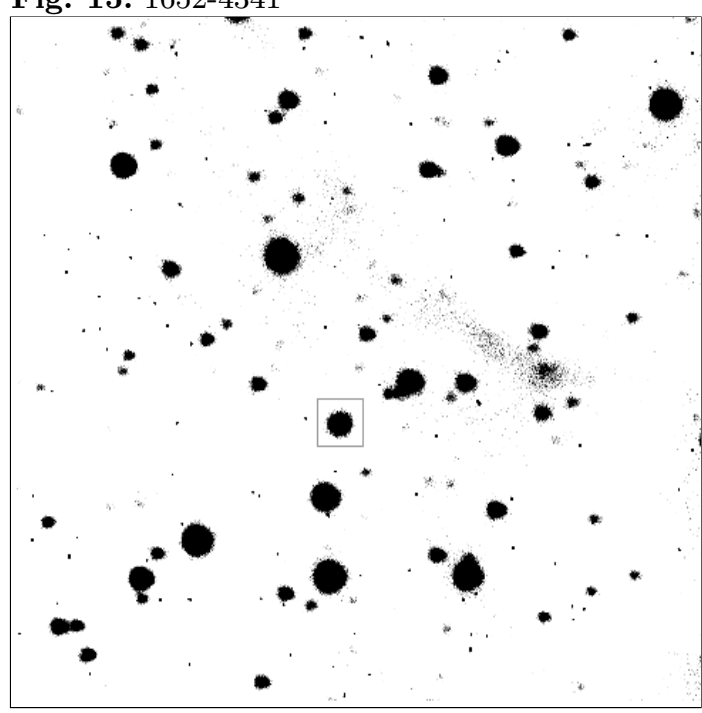

Fig. 16. 1708-4227

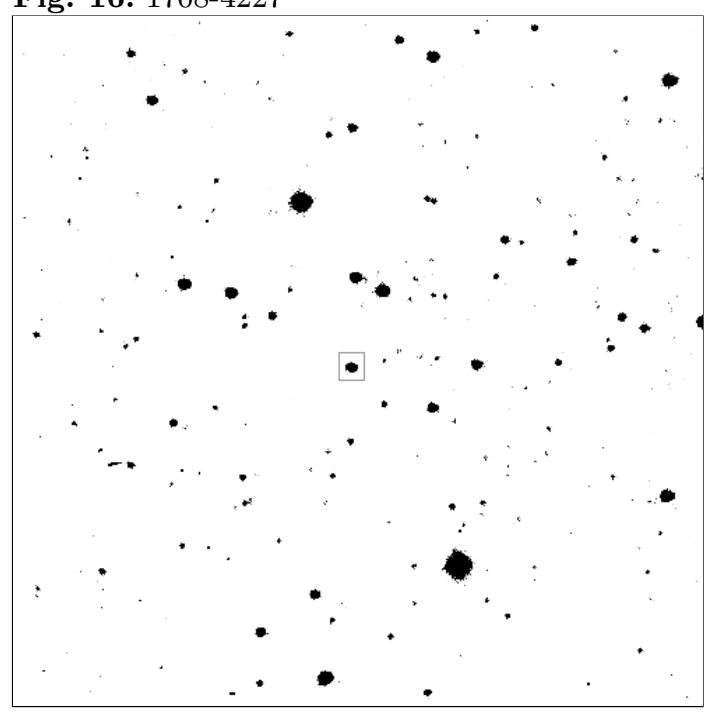




\section{B. Contour Plots}
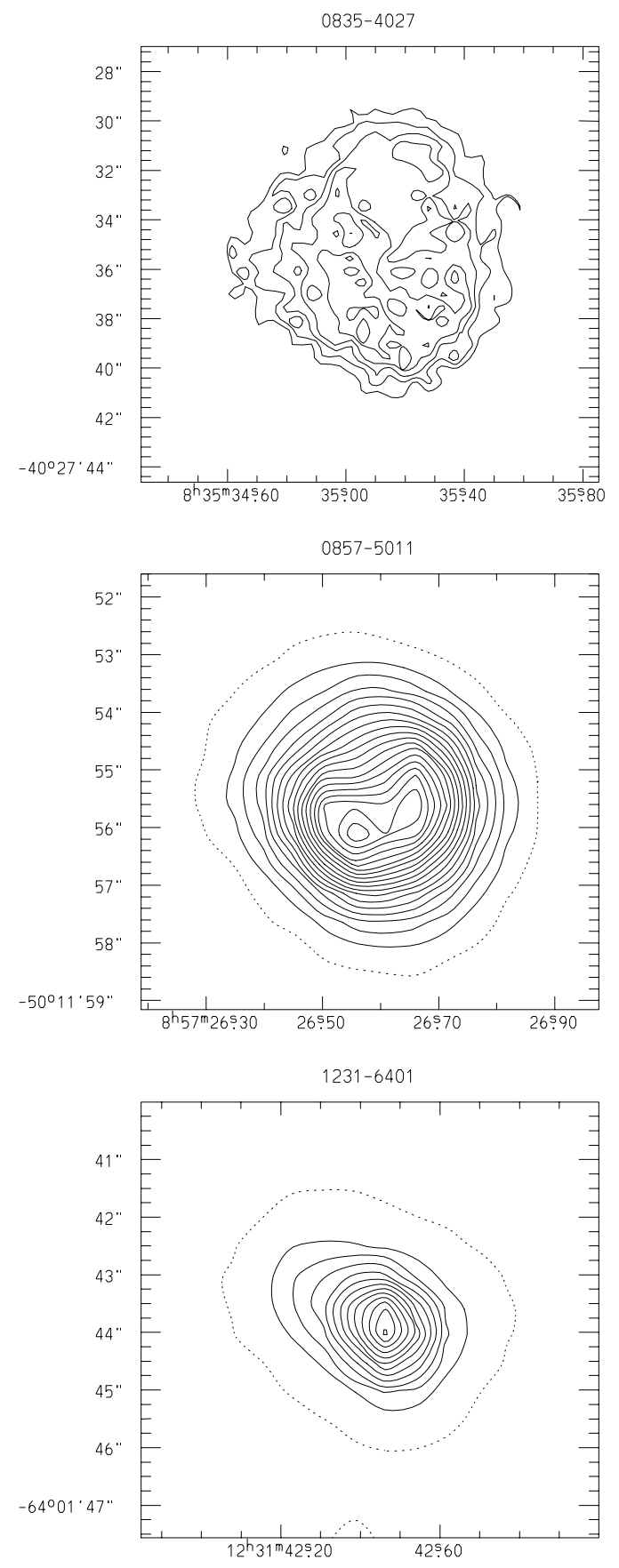

Top: the outer, lowest contour is at $50 \%$ of the peak; the stepsize is $10 \%$ of the peak.

Middle: the outer, lowest contour is at $5 \%$ of the peak; the stepsize is $5 \%$ of the peak.

Bottom: the outer, lowest contour is drawn at $2 \%$ of the peak; the stepsize is $8 \%$ of the peak.
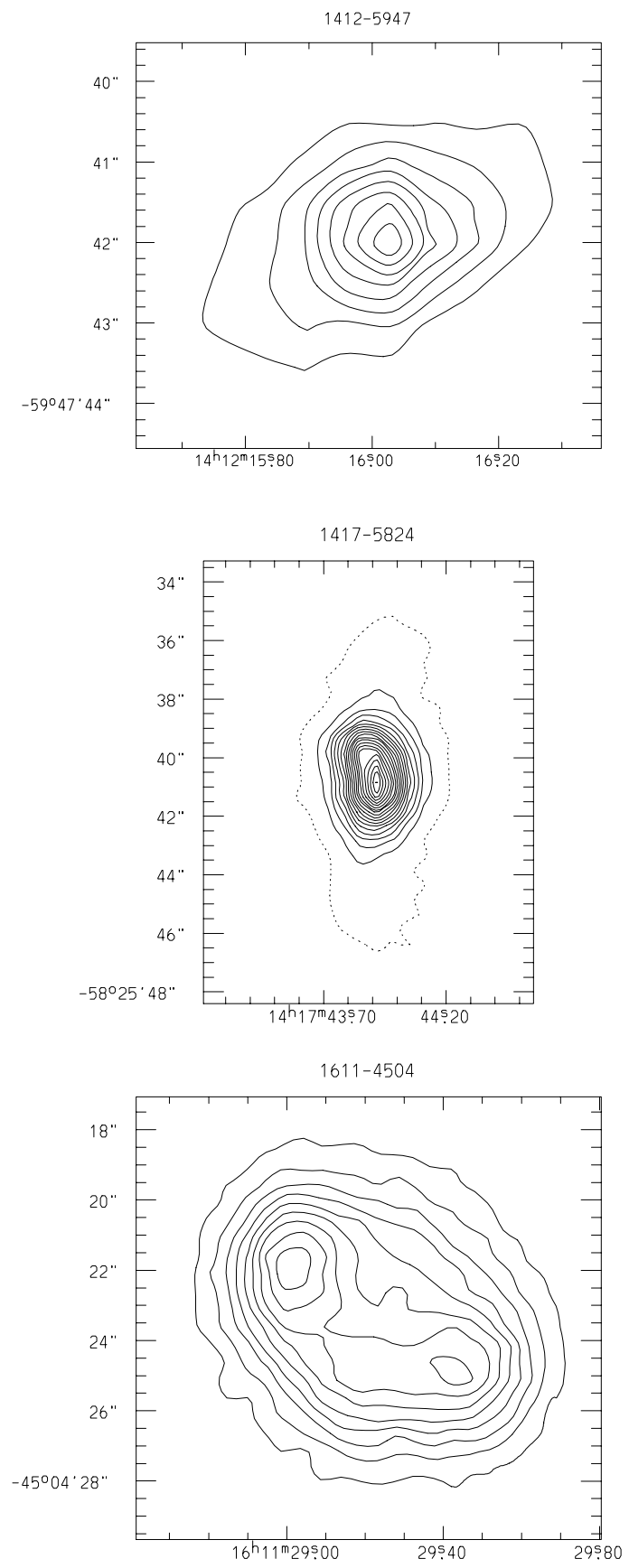

Top: the outer, lowest contour is at $20 \%$ of the peak; the stepsize is $10 \%$ of the peak.

Middle: the outer, lowest contour is at $5 \%$ of the peak; the stepsize is $5 \%$ of the peak

Bottom: the outer, lowest contour is at $10 \%$ of the peak; the stepsize is $10 \%$ of the peak. 


\section{Optical spectra}
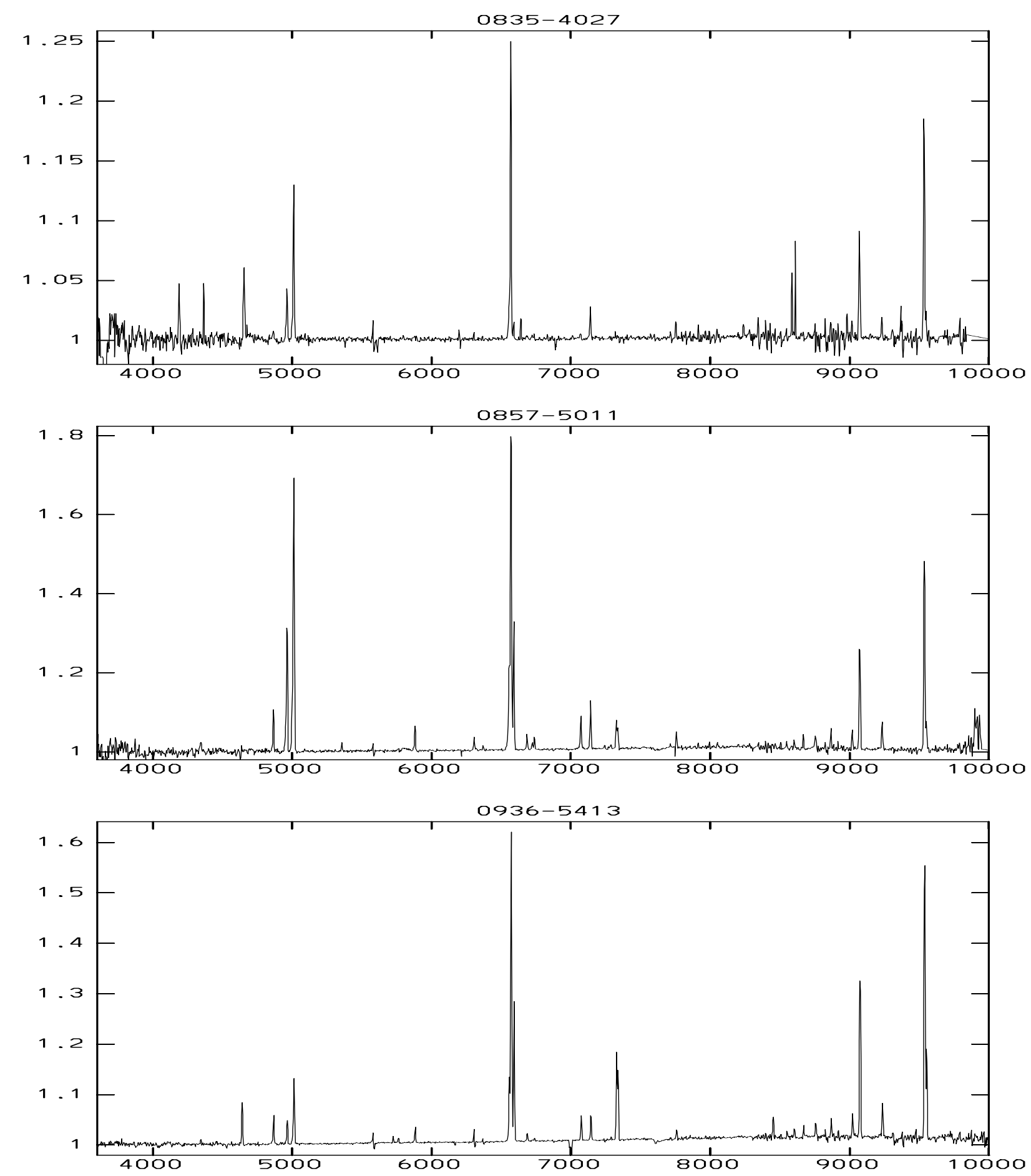

Spectra of IRAS-selected planetary nebula candidates.

Above every spectrum the name of the object is mentioned. $X$-axis: wavelength in angstroms from $3600 \AA$ to $10000 \AA$ $Y$-axis: $\log \left(10+I 10^{15}\right)$ in ergs $/ \mathrm{cm}^{2} / \mathrm{s} / \AA$ 

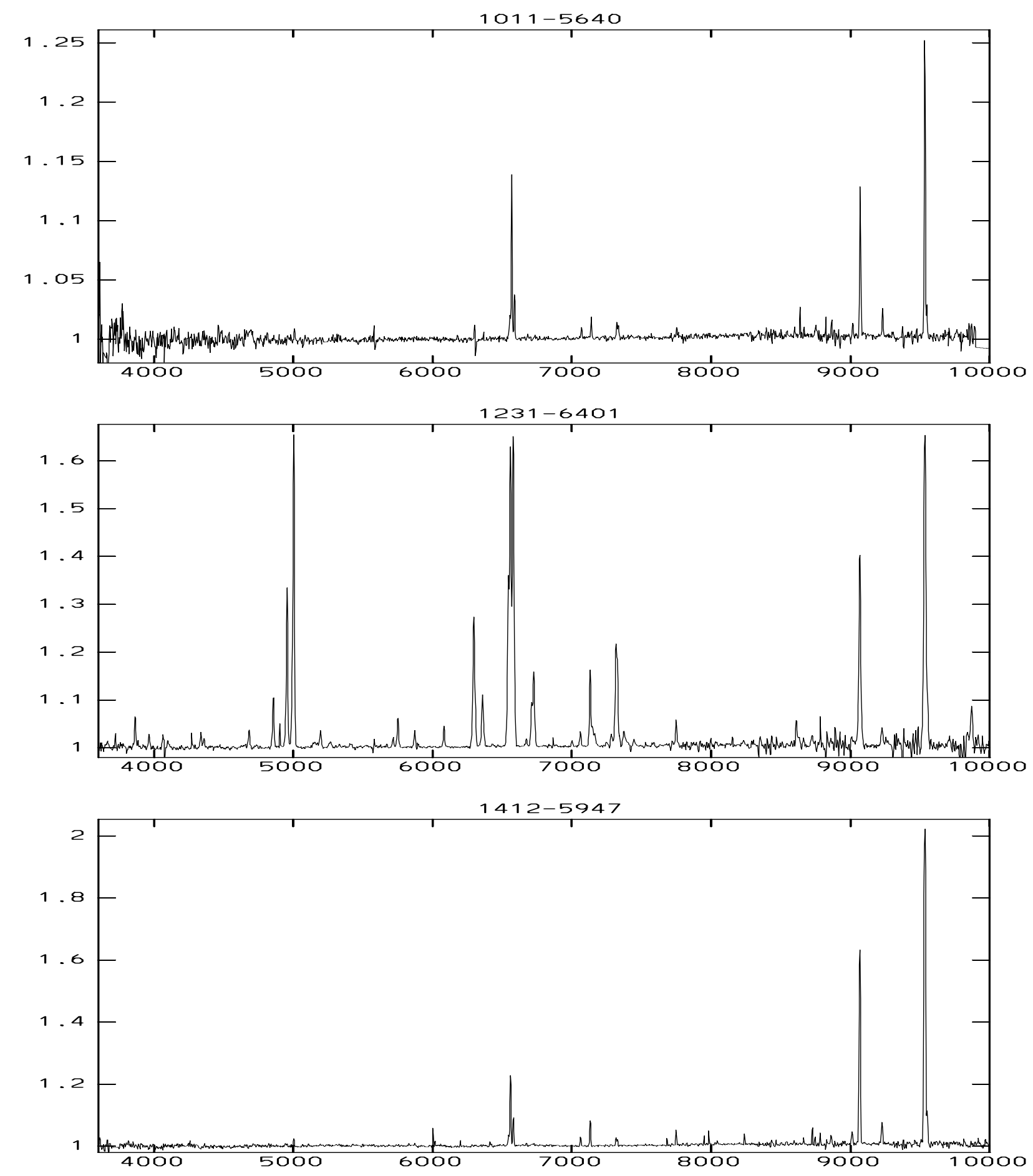

Spectra of IRAS-selected planetary nebula candidates. Above every spectrum the name of the object is mentioned. $X$-axis: wavelength in angstroms from $3600 \AA$ to $10000 \AA$ $Y$-axis: $\log \left(10+I 10^{15}\right)$ in ergs $/ \mathrm{cm}^{2} / \mathrm{s} / \AA$ 
$1417-5858$
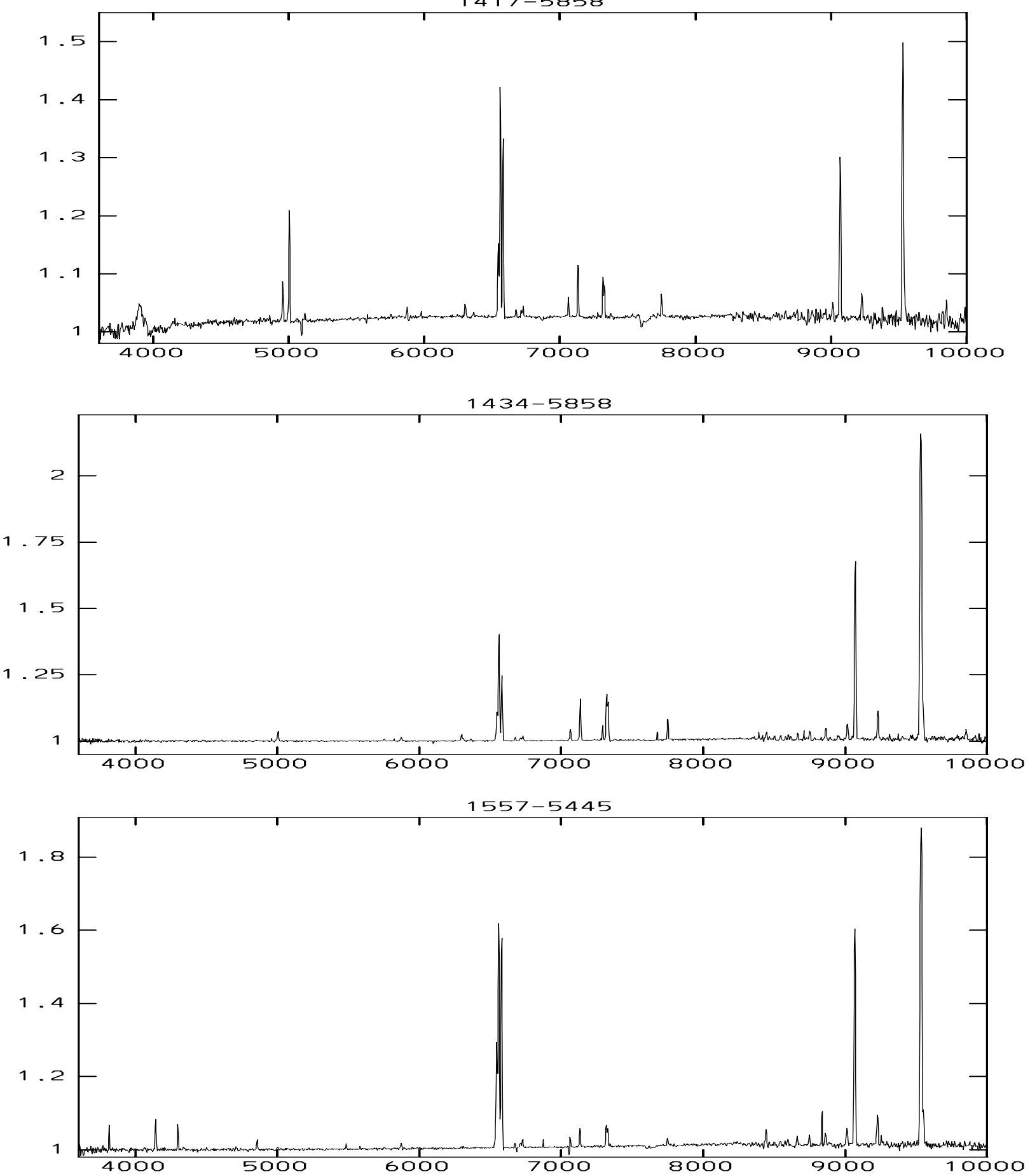

Spectra of IRAS-selected planetary nebula candidates.

Above every spectrum the name of the object is mentioned.

$X$-axis: wavelength in angstroms from $3600 \AA$ to $10000 \AA$

$Y$-axis: $\log \left(10+I 10^{15}\right)$ in $\mathrm{ergs} / \mathrm{cm}^{2} / \mathrm{s} / \AA$ 

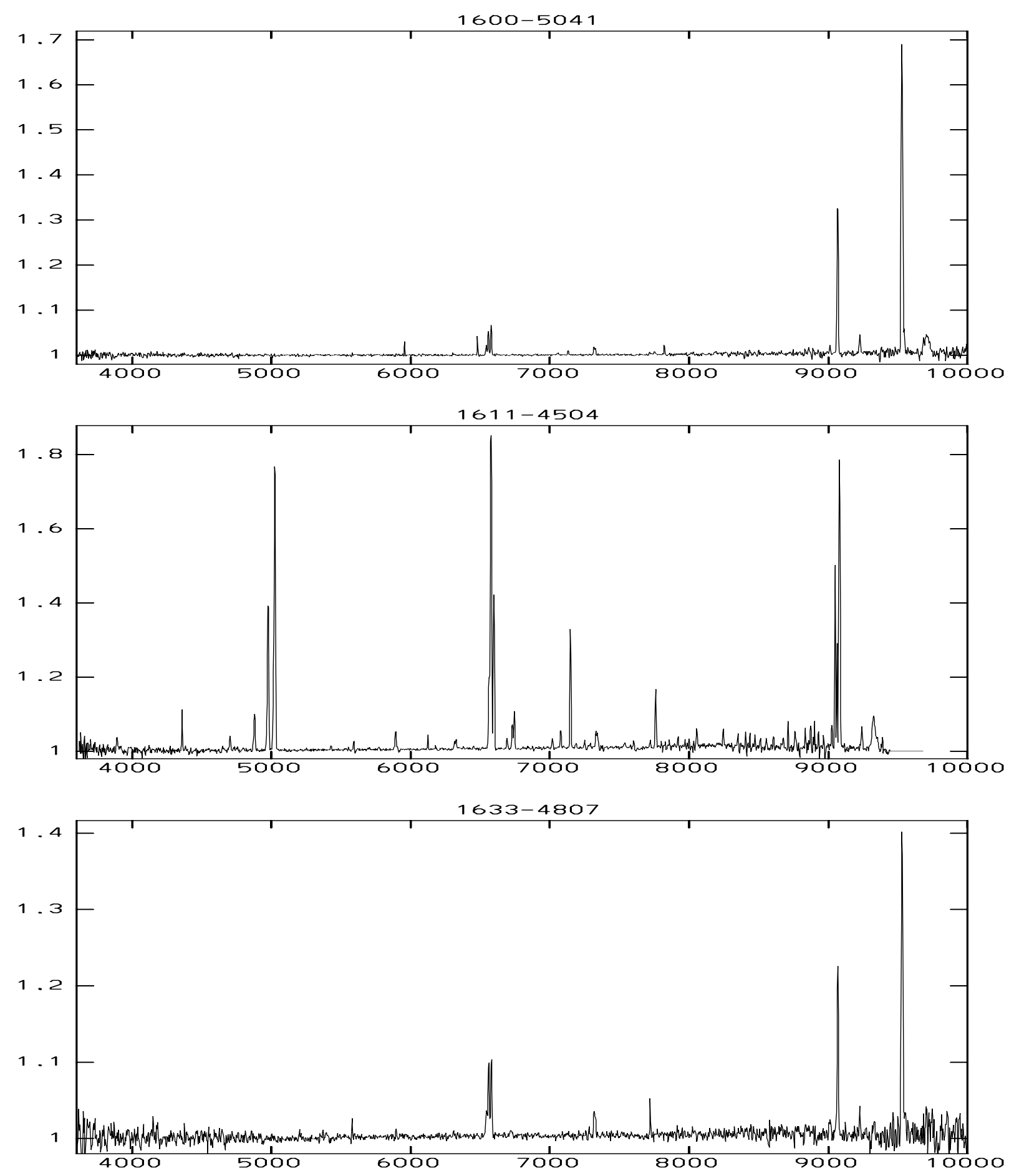

Spectra of IRAS-selected planetary nebula candidates. Above every spectrum the name of the object is mentioned. $X$-axis: wavelength in angstroms from $3600 \AA$ to $10000 \AA$ $Y$-axis: $\log \left(10+I 10^{15}\right)$ in ergs $/ \mathrm{cm}^{2} / \mathrm{s} / \AA$ 

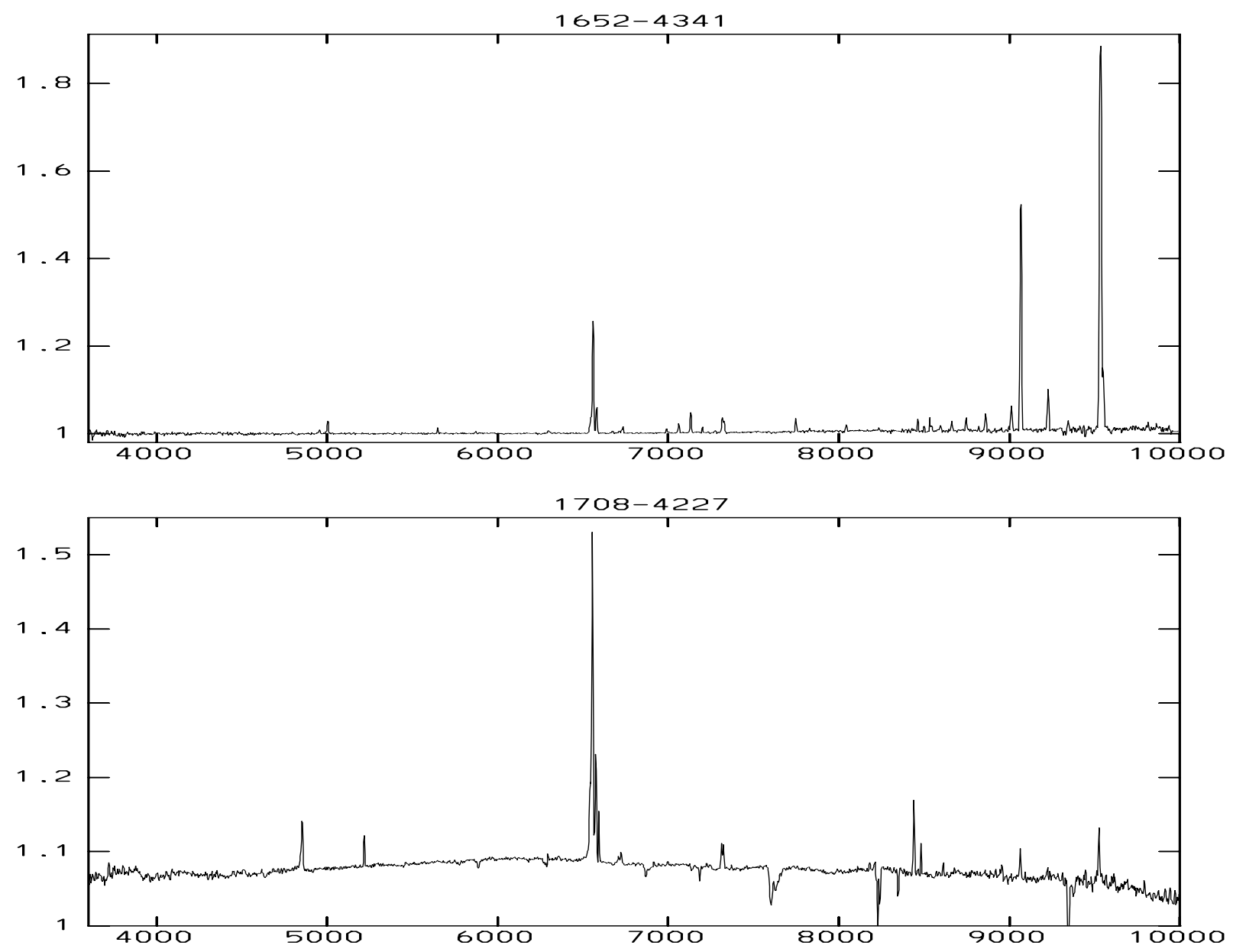

Spectra of IRAS-selected planetary nebula candidates.

Above every spectrum the name of the object is mentioned.

$X$-axis: wavelength in angstroms from $3600 \AA$ to $10000 \AA$

$Y$-axis: $\log \left(10+I 10^{15}\right)$ in ergs $/ \mathrm{cm}^{2} / \mathrm{s} / \AA$ 


\section{Line flux ratios}

Line fluxes corrected for interstellar extinction relative to $\mathrm{H} \beta$, [O III $] \lambda 5007$ or $\mathrm{H} \alpha$ depending upon which was available and well-determined. An estimate of the percentual uncertainty in the relative flux (err) is also given. Contaminated lines are indicated by a colon.

\begin{tabular}{|c|c|c|c|c|c|c|c|c|c|c|c|c|c|c|c|}
\hline$\overline{\lambda(\AA)}$ & Ident & $0835-$ & & $0857-5$ & & $0936-5$ & & 1011-5 & & $1231-6$ & & $1412-$ & & $1417-$ & \\
\hline & & Flux & err & Flux & err & Flux & err & Flux & err & Flux & err & Flux & err & Flux & err \\
\hline 3727 & {$[\mathrm{O} \mathrm{II}]$} & & & & & & & & & 58.1 & 11 & & & & \\
\hline 3869 & [Ne III] & & & & & & & & & 129.4 & 10 & & & & \\
\hline 3889 & $\mathrm{HeI}, \mathrm{HI}$ & & & & & & & & & 18.4 & 30 & & & & \\
\hline 3971 & $\mathrm{HI}$, [NeIII] & & & & & & & & & 58.2 & 10 & & & & \\
\hline 4069 & {$[\mathrm{~S} \mathrm{II}]$} & & & & & & & & & 53.9 & 10 & & & & \\
\hline 4102 & H I & & & & & & & & & 17.0 & 26 & & & & \\
\hline 4340 & H I & & & 85.9 & 26 & 39.2 & 17 & & & 47.7 & 10 & & & & \\
\hline 4363 & [O III] & & & & & & & & & 28.3 & 11 & & & & \\
\hline 4686 & $\mathrm{HeII}$ & & & & & & & & & 41.3 & 10 & & & & \\
\hline 4861 & H I & 5.9 & 28 & 100.0 & 10 & 100.0 & 10 & & & 100.0 & 10 & & & 6.9 & 16 \\
\hline 4959 & [O III] & 33.6 & 10 & 464.7 & 10 & 83.3 & 10 & & & 331.5 & 10 & 41.8 & 19 & 34.3 & 10 \\
\hline 5007 & [O III $]$ & 100.0 & 10 & 1341.7 & 10 & 263.0 & 10 & & & 986.1 & 10 & 100.0 & 10 & 100.0 & 10 \\
\hline 5200 & {$[\mathrm{~N} \mathrm{I}]$} & & & & & & & & & 29.6 & 10 & & & & \\
\hline 5309 & {$[\mathrm{Ca} \mathrm{V}]$} & & & & & & & & & 2.8 & 38 & & & & \\
\hline 5577 & {$\left[\begin{array}{ll}\mathrm{O} & \mathrm{I}\end{array}\right]$} & $2.7:$ & 17 & $3.9:$ & 22 & 10: & 10 & $51.8:$ & 12 & & & & & 0.7 & 43 \\
\hline 5755 & [N II] & & & & & 4.9 & 18 & & & 23.9 & 10 & & & 0.4 & 57 \\
\hline 5876 & $\mathrm{HeI}$ & & & 17.4 & 10 & 13.8 & 10 & & & 10.7 & 10 & & & 2.0 & 10 \\
\hline 6300 & [O I] & 0.4 & 33 & 4.9 & 10 & $3.6:$ & 12 & 13.2 & 11 & 121.8 & 10 & & & 1.5 & 14 \\
\hline 6312 & [S III] & & & & & & & & & 121.8 & 10 & & & & \\
\hline 6364 & {$[\mathrm{O} I]$} & 0.3 & 47 & 2.0 & 19 & 1.3: & 33 & $3.8:$ & 35 & 38.0 & 10 & & & 0.4 & 25 \\
\hline 6548 & [N II] & & & 42.3 & 10 & 35.3 & 10 & 19.8 & 14 & 119.7 & 10 & 11.5 & 16 & 5.1 & 10 \\
\hline 6563 & H I & 16.7 & 10 & 285.0 & 10 & 285.0 & 10 & 100.0 & 10 & 285.0 & 10 & 54.0 & 10 & 19.7 & 10 \\
\hline 6584 & [N II] & & & 50.7 & 10 & 77.4 & 10 & 26.0 & 10 & 321.4 & 10 & 20.6 & 10 & 13.6 & 10 \\
\hline 6678 & $\mathrm{H} \in \mathrm{I}$ & & & 4.2 & 10 & 3.2 & 10 & 3.3 & 29 & 2.7 & 12 & 1.2 & 27 & 0.4 & 14 \\
\hline 6716 & [S II] & & & 2.3 & 14 & 1.0 & 50 & 3.7 & 38 & 16.8 & 10 & 0.6 & 65 & 0.3 & 15 \\
\hline 6731 & [S II] & & & 3.2 & 10 & 1.1 & 29 & 3.1 & 26 & 40.7 & 10 & 1.0 & 27 & 0.6 & 11 \\
\hline 7006 & {$[\mathrm{Ar} \mathrm{V}]$} & & & & & & & & & 2.4 & 14 & & & & \\
\hline 7065 & $\mathrm{H} \in \mathrm{I}$ & 0.2 & 42 & 7.9 & 10 & 9.0 & 10 & 3.2 & 15 & 5.0 & 10 & 3.4 & 10 & 0.7 & 10 \\
\hline 7136 & [Ar III] & 0.9 & 10 & 11.1 & 10 & 7.6 & 10 & 6.4 & 10 & & & 9.0 & 10 & 1.8 & 10 \\
\hline 7178 & HeII & & & & & & & & & & & & & & \\
\hline 7281 & $\mathrm{HeI}$ & & & 1.7 & 66 & 0.5 & 40 & & & 4.2 & 10 & 0.8 & 83 & 0.1 & 22 \\
\hline 7323 & {$[\mathrm{O} \quad \mathrm{II}]$} & & & 5.5 & 10 & 28.3 & 10 & 3.0 & 11 & 73.1 & 10 & 2.5 & 10 & 1.2 & 10 \\
\hline 7332 & {$[\mathrm{O} I 1]$} & & & 5.1 & 10 & 21.9 & 10 & 4.3 & 20 & 73.1 & 10 & 1.5 & 10 & 0.8 & 10 \\
\hline 7751 & [Ar III] & 0.3 & 12 & $2.6:$ & 10 & 2.4 & 10 & 1.4 & 15 & 6.7 & 10 & 2.6 & 10 & 0.4 & 10 \\
\hline 8046 & {$[\mathrm{Cl} I \mathrm{~V}]$} & & & & & & & & & & & & & & \\
\hline 8237 & $\mathrm{HeII}$ & 0.2 & 17 & & & & & & & 1.1 & 19 & & & & \\
\hline 8446 & $\mathrm{OI}, \mathrm{HI}$ & & & & & 37.3 & 10 & & & & & & & & \\
\hline 8467 & $\mathrm{H} \mathrm{I}$ & & & & & & & & & & & & & & \\
\hline 8502 & H I & & & 0.6 & 13 & 0.8 & 16 & & & & & & & & \\
\hline 8545 & H I & & & 0.5 & 13 & 1.2 & 11 & & & & & 0.4 & 30 & & \\
\hline 8579 & [Cl II] & & & & & & & & & & & & & & \\
\hline 8598 & $\mathrm{HI}$ & & & 0.8 & 10 & 1.4 & 10 & & & & & 0.3 & 15 & & \\
\hline 8665 & H I & & & 1.0 & 10 & 1.2 & 10 & & & & & 0.3 & 12 & & \\
\hline 8750 & $\mathrm{H} \mathrm{I}$ & & & 1.4 & 14 & 1.7 & 10 & 0.3 & 22 & & & 0.5 & 10 & & \\
\hline 8863 & $\mathrm{H} \mathrm{I}$ & 0.1 & 11 & 1.3 & 10 & 1.7 & 10 & 0.7 & 10 & & & 0.5 & 10 & & \\
\hline 9015 & H I & 0.1 & 11 & 1.3 & 10 & 2.1 & 10 & 0.5 & 11 & & & 0.7 & 10 & 0.1 & 21 \\
\hline 9069 & [S III] & 0.6 & 10 & 9.2 & 10 & 19.8 & 10 & 5.0 & 10 & 47.3 & 10 & 18.1 & 10 & 1.2 & 10 \\
\hline 9229 & $\mathrm{HI}$ & 0.1 & 12 & 1.6 & 10 & 3.0 & 10 & 0.7 & 10 & 3.4 & 12 & 1.0 & 10 & 0.1 & 10 \\
\hline 9532 & [S III] & 1.2 & 10 & 21.8 & 10 & 45.8 & 10 & 10.3 & 10 & 114.9 & 10 & 50.6 & 10 & 2.7 & 10 \\
\hline 9546 & $\mathrm{HI}$ & 0.1 & 10 & 1.7 & 10 & 8.0 & 10 & 0.9 & 10 & & & 0.7 & 10 & & \\
\hline
\end{tabular}


Line fluxes corrected for interstellar extinction relative to $\mathrm{H} \beta$, [O III] $\lambda 5007$ or $\mathrm{H} \alpha$ depending upon which was available and well-determined. An estimate of the percentual uncertainty in the relative flux (err) is also given.

Contaminated lines are indicated by a column.

\begin{tabular}{|c|c|c|c|c|c|c|c|c|c|c|c|c|c|c|c|}
\hline$\lambda(\AA)$ & Ident & $1434-$ & & $1557-5$ & & $1600-5$ & & $1611-4$ & & $1633-$ & & $1652-4$ & & $1708-4$ & \\
\hline & & Flux & err & Flux & err & Flux & err & Flux & err & Flux & err & Flux & err & Flux & err \\
\hline$\overline{3727}$ & {$[\mathrm{O} \mathrm{II}]$} & & & & & & & & & & & & & & \\
\hline 3869 & [Ne III] & & & & & & & & & & & & & & \\
\hline 3889 & $\mathrm{He}$, H I & & & & & & & & & & & & & & \\
\hline 3971 & $\mathrm{HI},[\mathrm{NeIII}]$ & & & & & & & & & & & & & & \\
\hline 4069 & [S II] & & & & & & & & & & & & & & \\
\hline 4102 & H I & & & & & & & & & & & & & & \\
\hline 4340 & H I & & & 91.6 & 29 & & & & & & & & & & \\
\hline 4863 & [O III] & & & & & & & & & & & & & & \\
\hline 4686 & $\mathrm{H} \in \mathrm{II}$ & & & & & & & & & & & & & & \\
\hline 4861 & H I & $0.07:$ & 73 & 100.0 & 10 & & & 100.0 & 10 & & & & & 100.0 & 10 \\
\hline 4959 & [O III] & (33) & & $(10.7)$ & & & & 451.7 & 10 & & & 30.3 & 13 & & \\
\hline 5007 & [O III] & 100.0 & 10 & 32.1 & 46 & & & 1360.3 & 10 & & & 100.0 & 10 & & \\
\hline 5200 & {$[\mathrm{~N} \mathrm{I}]$} & & & & & & & & & & & & & & \\
\hline 5309 & {$[\mathrm{Ca} \mathrm{V}]$} & 4.1: & 53 & & & & & & & & & & & & \\
\hline 5577 & {$[\mathrm{O} I]$} & & & & & & & 5.5 & 30 & 78.9 & 17 & & & & \\
\hline 5755 & {$[\mathrm{~N}$ II] } & $3.9:$ & 38 & 6.4 & 43 & & & 2.1 & 75 & & & & & & \\
\hline 5876 & $\mathrm{HeI}$ & 5.1: & 14 & 12.6 & 12 & & & 12.0 & 11 & & & 2.1 & 29 & & \\
\hline 6300 & {$[\mathrm{O} \mathrm{I}]$} & 3.3: & 10 & & & & & 2.1 & 32 & & & 1.7 & 37 & & \\
\hline 6312 & [S III] & 1.1: & 20 & & & & & 3.6 & 22 & & & & & & \\
\hline 6364 & {$[\mathrm{O} I]$} & 1.1: & 43 & & & & & 0.7 & 79 & & & & & & \\
\hline 6548 & [N II] & 13.0: & 10 & 104.3 & 10 & 40.9 & 10 & 26.4 & 10 & 44.6 & 12 & 9.5 & 10 & 215.6 & 10 \\
\hline 6563 & H I & $56.3:$ & 10 & 285.0 & 10 & 100.0 & 10 & 285.0 & 10 & 100.0 & 10 & 37.0 & 10 & 285.0 & 10 \\
\hline 6584 & [N II] & $27.8:$ & 10 & 261.5 & 10 & 115.6 & 10 & 76.1 & 10 & 104.2 & 10 & 7.4 & 10 & & \\
\hline 6678 & $\mathrm{HeI}$ & 0.8 & 10 & 2.6 & 15 & & & 3.2 & 19 & & & 0.6 & 19 & & \\
\hline 6716 & [S II] & $0.9:$ & 26 & 2.4 & 18 & & & 7.0 & 10 & 7.5 & 34 & 0.5 & 18 & 4.8 & 22 \\
\hline 6731 & [S II] & 1.3: & 10 & 4.0 & 10 & & & 11.3 & 10 & 2.3 & 95 & 1.6 & 10 & 7.7 & 11 \\
\hline 7006 & {$[\mathrm{ArV}]$} & & & & & & & 2.1 & 20 & & & 0.3 & 36 & & \\
\hline 7065 & $\mathrm{He} \mathrm{I}$ & 1.9: & 10 & 2.6 & 10 & & & 3.6 & 11 & & & 1.2 & 10 & & \\
\hline 7136 & [Ar III] & $7.2:$ & 10 & 6.3 & 10 & 7.4 & 23 & 31.8 & 10 & & & 2.5 & 10 & & \\
\hline 7178 & He II & & & & & & & 1.3 & 106 & & & & & & \\
\hline 7281 & $\mathrm{HeI}$ & & & & & & & 2.0 & 15 & & & 0.3 & 18 & & \\
\hline 7323 & {$[\mathrm{O} \quad \mathrm{II}]$} & $7.0:$ & 10 & 6.4 & 10 & 11.0 & 11 & 3.4 & 28 & & & 1.6 & 10 & 15.0 & 10 \\
\hline 7332 & [O II] & $5.0:$ & 10 & 4.8 & 10 & 9.3 & 14 & & & & & 1.2 & 10 & 13.4 & 10 \\
\hline 7751 & [Ar III] & 1.7: & 10 & 1.5 & 10 & & & 8.1 & 10 & & & 0.9 & 10 & & \\
\hline 8046 & [Cl IV] & & & & & & & & & & & 0.4 & 10 & & \\
\hline 8237 & $\mathrm{He}$ II & & & & & & & & & & & 0.1 & 13 & & \\
\hline 8446 & {$[\mathrm{O} \mathrm{I}], \mathrm{H} \mathrm{I}$} & 0.06 & 10 & 2.8 & 10 & & & & & & & & & 38.8 & 10 \\
\hline 8467 & H I & $0.3:$ & 10 & & & & & & & & & 0.3 & 10 & & \\
\hline 8502 & H I & 0.1 : & 16 & & & & & 0.8 & 22 & & & 0.2 & 10 & & \\
\hline 8545 & H I & $0.1:$ & 12 & 0.6 & 15 & & & 1.0 & 19 & & & 0.2 & 10 & & \\
\hline 8579 & [Cl II] & 0.1 : & 13 & 0.5 & 14 & & & & & & & & & & \\
\hline 8598 & $\mathrm{H} \mathrm{I}$ & 0.1 : & 10 & 0.7 & 11 & & & 1.1 & 15 & & & 0.1 & 10 & & \\
\hline 8665 & $\mathrm{H} \mathrm{I}$ & $0.2:$ & 10 & 0.8 & 10 & & & 0.6 & 25 & & & 0.2 & 10 & & \\
\hline 8750 & $\mathrm{H} \mathrm{I}$ & $0.3:$ & 10 & 1.0 & 10 & & & 2.1 & 32 & & & 0.2 & 10 & & \\
\hline 8863 & H I & $0.3:$ & 10 & 1.0 & 10 & & & 1.7 & 30 & & & 0.3 & 10 & & \\
\hline 9015 & H I & 0.3 : & 10 & 1.1 & 10 & 1.1 & 10 & 1.0 & 10 & & & 0.3 & 10 & & \\
\hline 9069 & [S III] & $6.0:$ & 10 & 25.4 & 10 & 26.5 & 10 & 41.5 & 10 & 18.4 & 10 & 4.7 & 10 & 11.0 & 10 \\
\hline 9229 & $\mathrm{HI}$ & $0.4:$ & 10 & 1.9 & 10 & 2.0 & 10 & & & 2.2 & 10 & 0.5 & 10 & & \\
\hline 9532 & [S III] & 19.9: & 10 & 57.6 & 10 & 82.0 & 10 & & & 42.4 & 10 & 12.7 & 10 & 18.2 & 10 \\
\hline 9546 & $\mathrm{H} \mathrm{I}$ & $0.4:$ & 10 & 1.1 & 10 & 3.3 & 10 & & & 2.7 & 15 & 0.5 & 10 & & \\
\hline
\end{tabular}

